The Journal of Nonlinear $\mathbf{S}$ ciences and Applications http://www.tjnsa.com

\title{
SOME REMARK ON THE NONEXISTENCE OF POSITIVE SOLUTIONS FOR SOME $a, P$-LAPLACIAN SYSTEMS
}

\author{
M. ALIMOHAMMADY ${ }^{1 *}$ AND M. KOOZEGAR ${ }^{2}$
}

ABSTRACT. This paper deals with nonexistence result for positive solution in $\mathrm{C}^{1}(\bar{\Omega})$ to the following reaction-diffusion system

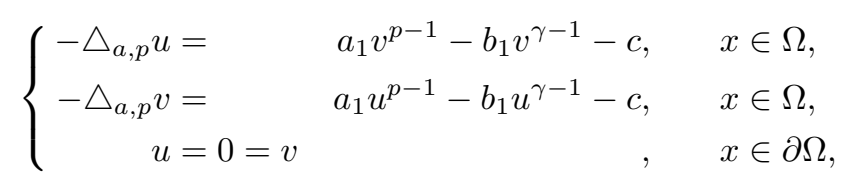

where $\triangle_{a, p}$ denotes the $a, p$-Laplacian operator defined by $\triangle_{a, p} z=\operatorname{div}(a \mid$ $\left.\left.\nabla z\right|^{p-2} \nabla z\right) ; p>1, \gamma(>p) ; a_{1}, b_{1}$ and $c$ are positive constant, $\Omega$ is a smooth bounded domain in $\mathbb{R}^{N}(N \geq 1)$ with smooth boundary and $a(x) \in L^{\infty}(\Omega)$, $a(x) \geq a_{0}>0$ for all $x \in \Omega$.

\section{INTRODUCTION AND PRELIMINARIES}

In this note, we first consider a nonexistence result for positive solution in $\mathrm{C}^{1}(\bar{\Omega})$ to the following reaction-diffusion system

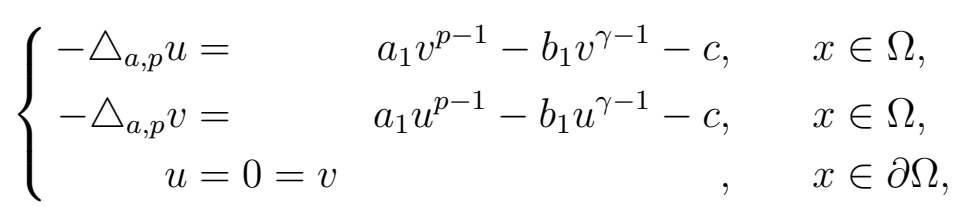

where $\triangle_{a, p}$ denotes the $p, a$-Laplacian operator defined by $\triangle_{a, p} z=\operatorname{div}\left(a|\nabla z|^{p-2}\right.$ $\nabla z) ; p>1, \gamma(>p) ; a_{1}, b_{1}$ and $c$ are positive constant, $\Omega$ is a smooth bounded domain in $\mathbb{R}^{N}(N \geq 1)$ with smooth boundary and $a(x) \in L^{\infty}(\Omega), a(x) \geq a_{0}>0$

Date: Received: 10 August 2008; Accepted: 10 September 2008.

* Corresponding author.

2000 Mathematics Subject Classification. Primary 35P20, Secondary 35J70; 35BJ20.

Key words and phrases. p,a-Laplacian; nonexistence; positive solution; reaction-diffusion systems. 
for all $x \in \Omega$.

We first show that if $a_{1} \leq \lambda_{1}$, where $\lambda_{1}$ is the first eigenvalue of $-\triangle_{p, a}$ with Dirichlet boundary condition, then (1.1) has no positive solutions. next we consider the system

$$
\left\{\begin{aligned}
-\triangle_{a, p} u & =\lambda f(v), & & x \in \Omega, \\
-\triangle_{a, p} v & =\mu g(u), & & x \in \Omega, \\
u & =0=v, & & x \in \partial \Omega .
\end{aligned}\right.
$$

where $\Omega$ is a smooth bounded domain in $\mathbb{R}^{N}(N \geq 1), \partial \Omega$ is its smooth boundary and $\lambda, \mu$ are positive parameters. Let $f, g:[0, \infty) \rightarrow R$ be continuous and assume that there exist positive numbers $K_{i}$ and $M_{i}, i=1,2$ such that

$$
f(v) \geq K_{1} v^{p-1}+M_{1}(v \geq 0),
$$

and

$$
g(u) \geq K_{2} u^{p-1}+M_{2}(u \geq 0),
$$

and $a: \Omega \rightarrow \mathbb{R}^{+}$satisfy certain conditions.

We discus a non existence result when $\lambda \mu$ is large.

Definition 1.1. A pair of nonnegative functions $(u, v)$ in $\mathrm{W}_{0}^{1, \mathrm{p}}(\Omega) \times \mathrm{W}_{0}^{1, \mathrm{p}}(\Omega)$ are called a weak solution of (1.2) if they satisfies

$$
\int_{\Omega} a|\nabla u|^{p-2} \nabla u \nabla w d x=\int_{\Omega}[\lambda f(v)] w d x
$$

and

$$
\int_{\Omega} a|\nabla v|^{p-2} \nabla v \nabla w d x=\int_{\Omega}[\mu g(u)] w d x
$$

for all test function $w \in \mathrm{W}_{0}^{1, \mathrm{p}}(\Omega)$.

In the case $a=1$ when $p=2$, system (1.2) studied by Dalmaso [5]. For existence results of positive solutions for (1.2) see [3, 4, 6]. For corresponding result in the single equations case see [2, 8] for (1.1) and [7] for (1.2), (for the case $a=1$ ) and in [1] Afrouzi and Rasouli studied the system (1.1), (1.2) for the case $a=1$.

\section{Non-existence Results}

In this section we state our main results. To prove the non-existence results we use some estimates on the first eigenvalue of $-\triangle_{p, a}$ with Dirichlet boundary conditions.

Theorem 2.1. let $q$ be such that $\frac{1}{p}+\frac{1}{q}=1$. Then for $a_{1} \leq \lambda_{1}$, (1.1) has no positive solution. 
Proof. On the contrary, there exists a positive solution $(u, v)$ of (1.1). Since for any $\mathrm{w} \in \mathrm{C}_{0}^{\infty}(\Omega)$ we have

SO

$$
\int_{\Omega} a|\nabla u|^{p-2} \nabla u \nabla w d x=\int_{\Omega}\left[a_{1} v^{p-1}-b_{1} v^{\gamma-1}-c\right] w d x
$$

and since $b_{1}>0$, then

$$
\int_{\Omega} a|\nabla u|^{p} d x=\int_{\Omega}\left[a_{1} v^{p-1}-b_{1} v^{\gamma-1}-c\right] u d x
$$

$$
\int_{\Omega} a|\nabla u|^{p} d x \leq \int_{\Omega}\left[a_{1} v^{p-1}-c\right] u d x
$$

But

$$
\int_{\Omega} a|\nabla u|^{p} d x \geq \lambda_{1} \int_{\Omega}|u|^{p} d x=\lambda_{1} \int_{\Omega} u^{p} d x
$$

since $\lambda_{1}=\inf _{z \in \mathrm{W}_{0}^{1, \mathrm{p}}(\Omega)} \frac{\int_{\Omega} a|\nabla z|^{p} d x}{\int_{\Omega}|z|^{p} d x}$ is the first eigenvalue of $-\triangle_{a, p}$ with Dirichlet boundary condition. Combining (2.1), (2.2), we obtain

$$
\lambda_{1} \int_{\Omega} u^{p} d x \leq a_{1} \int_{\Omega} u v^{p-1} d x-c \int_{\Omega} u d x
$$

Similarly, we obtain

$$
\lambda_{1} \int_{\Omega} v^{p} d x \leq a_{1} \int_{\Omega} v u^{p-1} d x-c \int_{\Omega} v d x
$$

But recall that $a b \leq \frac{a^{p}}{p}+\frac{b^{q}}{q}$ if $\frac{1}{p}+\frac{1}{q}=1$. Taking $a=u, b=v^{p-1}$ and $a=v, b=u^{p-1}$, we see respectively that $u v^{p-1} \leq \frac{u^{p}}{p}+\frac{v^{p}}{q}$ and $v u^{p-1} \leq \frac{v^{p}}{p}+\frac{u^{p}}{q}$. Thus adding (2.3) and (2.4),

$$
\begin{gathered}
\lambda_{1} \int_{\Omega} u^{p} d x+\lambda_{1} \int_{\Omega} v^{p} d x \\
\leq a_{1} \int_{\Omega} u v^{p-1} d x+a_{1} \int_{\Omega} v u^{p-1} d x-c \int_{\Omega}(u+v) d x \\
<a_{1} \int_{\Omega} u v^{p-1} d x+a_{1} \int_{\Omega} v u^{p-1} d x \\
\leq a_{1} \int_{\Omega}\left[\frac{u^{p}}{p}+\frac{v^{p}}{q}\right] d x+a_{1} \int_{\Omega}\left[\frac{v^{p}}{p}+\frac{u^{p}}{q}\right] d x \\
=a_{1}\left(\frac{1}{p}+\frac{1}{q}\right) \int_{\Omega} u^{p} d x+a_{1}\left(\frac{1}{p}+\frac{1}{q}\right) \int_{\Omega} v^{p} d x \\
=a_{1} \int_{\Omega}\left[u^{p}+v^{p}\right] d x .
\end{gathered}
$$

This implies that

$$
\left(\lambda_{1}-a_{1}\right) \int_{\Omega}\left[u^{p}+v^{p}\right] d x<0
$$


which is contradiction if $a_{1} \leq \lambda_{1}$. Thus (1.1) has no positive solutions for $a_{1} \leq \lambda_{1}$.

Now we consider the system (1.2) and we would establish the following.

Theorem 2.2. Let (1.3)-(1.4) hold. Then the system (1.2) has no positive solution if $\lambda \mu>\frac{\lambda_{1}^{2}}{K_{1} K_{2}}$.

Proof. Suppose $u>0$ and $v>0$ be $\mathrm{C}^{1}(\bar{\Omega})$ functions such that $(u, v)$ is a solution of (1.2). We proceed our proof by arriving to a contradiction. Multiplying the first equation in (1.2) by a positive eigenfunction say $\phi_{1}$ corresponding to $\lambda_{1}$, we obtain

and hence using (1.3),

$$
-\int_{\Omega} \triangle_{a, p} u \phi_{1} d x=\int_{\Omega} \lambda f(v) \phi_{1} d x
$$

That is

$$
-\int_{\Omega} \triangle_{a, p} u \phi_{1} d x \geq \int_{\Omega} \lambda\left(K_{1} v^{p-1}+M_{1}\right) \phi_{1} d x .
$$

$$
\int_{\Omega} u^{p-1} \lambda_{1} \phi_{1} d x \geq \int_{\Omega} \lambda\left(K_{1} v^{p-1}+M_{1}\right) \phi_{1} d x .
$$

Similarly using the second equation in (1.2) and (1.4),

$$
\int_{\Omega} v^{p-1} \lambda_{1} \phi_{1} d x \geq \int_{\Omega} \mu\left(K_{2} u^{p-1}+M_{2}\right) \phi_{1} d x .
$$

Combining (2.5) and (2.6),

$$
\begin{aligned}
\int_{\Omega}\left[\lambda_{1}-(\lambda \mu) \frac{K_{1} K_{2}}{\lambda_{1}}\right] v^{p-1} \phi_{1} d x \geq \int_{\Omega} \mu\left[K_{2} u^{p-1}+\right. & \left.M_{2}+\lambda \frac{M_{1} K_{2}}{\lambda_{1}}-K_{2} u^{p-1}\right] \phi_{1} d x \\
= & \int_{\Omega} \mu\left[\lambda \frac{K_{2} M_{1}}{\lambda_{1}}+M_{2}\right] \phi_{1} d x(2.7)
\end{aligned}
$$

This clearly require $\lambda \mu \leq \frac{\lambda_{1}^{2}}{K_{1} K_{2}}$. This completes the proof.

\section{REFERENCES}

1. G. A. Afrouzi, S. H. Rasouli, A remark on the nonexistence of positive solutions for some p-Laplacian systems, Global J. Pure. Appl. Math. 1/2(2005), 197-201. 1

2. G. A. Afrouzi, S. H. Rasouli, Population models involving the p-Laplacian with indefinite weight and constant yeild harvesting. , chaos, Solutions and Fractals, 31(2007), 404-408. 1

3. L. Boccardo, D. G. Figueiredo, Some remarks on a system of quasilinear elliptic equations. , Nonl. Diff. Eqns. Appl. 9(2002), 231-240. 1

4. P. Clement, J. Fleckinger, E. Mitidieri, F. de Thelin, Existence of positive solutions for a nonvariational quasilinear elliptic system., J. Diff. Eqns. 166(2000), 455-477. 1

5. R. Dalmasso, Existence and uniqueness of positive solutions of a semilinear elliptic system., Nonl. Anal. 39(2000), 559-568. 1 
6. A. Djellit, S. Tas, On some nonlinear elliptic systems., Nonl. Anal. 59(2004), 675-706. 1

7. D. D. Hai, On a cllas of sublinear quasilinear elliptic problems. Proc. Amer. Math. Soc. 131(2003), 2409-2414. 1

8. S. Oruganti, J. Shi, R. Shivaji, Logistic equation with the p-Laplacian and constant yeild harvesting., Applied. Anal. 9(2004), 723-727. 1

1 Islamic Azad University, BRAnCH NoOR, Iran

E-mail address: m.kaleji@yahoo.com

2 Department of Mathematics, University of Mazandaran, Babolsar 47416 1468, IRAN.

E-mail address: amohsen@umz.ac.ir 